\title{
Near field modal noise reduction using annealed optical fiber
}

\author{
Steve N. Osterman ${ }^{* a, b}$, Gabriel G. Ycas ${ }^{\mathrm{c}}$, Chelsea L. Donaldson ${ }^{\mathrm{d}}$, Scott A. Diddams ${ }^{\mathrm{c}, \mathrm{e}}$, \\ Suvrath Mahadevan ${ }^{\mathrm{f}, \mathrm{g}}$, Lawrence W. Ramsey ${ }^{\mathrm{f}, \mathrm{g}}$ and Peter P. Plavchan ${ }^{\mathrm{h}, \mathrm{i}}$
}

${ }^{\mathrm{a}}$ Johns Hopkins University Applied Physics Laboratory, Laurel, MD, USA; ${ }^{\mathrm{b}}$ Center for Astrophysics and Space Astronomy, University of Colorado, Boulder, CO, USA; ${ }^{\mathrm{c}}$ Time and Frequency Division, National Institute of Standards and Technology, Boulder, CO, USA; ${ }^{\mathrm{d}}$ Southwest Research Institute, Boulder, CO, USA $;{ }^{\mathrm{e}}$ Joint Institute for Laboratory Astrophysics, University of Colorado, Boulder, CO, USA $;{ }^{\mathrm{f}}$ Department of Astronomy and Astrophysics, Pennsylvania State University, University Park, PA, USA; ${ }^{\mathrm{g}}$ Center for Exoplanets and Habitable Worlds, Pennsylvania State University,

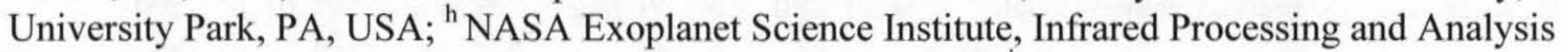
Center, California Institute of Technology, Pasadena, CA, USA; ${ }^{\mathrm{i}}$ Department of Physics, Astronomy and Material Science, Missouri State University, Springfield, MO, USA.

\begin{abstract}
Incomplete and unstable mode population has long complicated the application of optical fiber for transferring star and calibration light to high precision spectrographs. The need for improved precision calibrators in support of radial velocity planet surveys has led to the introduction of coherent wavelengths sources using single mode fibers that are then coupled into multi-mode fibers, further exacerbating this problem. We explore mode scrambling in annealed optical fiber with and without agitation, as compared to that obtained using octagonal fiber and using an integrating sphere. We observe improved scrambling with annealed fibers compared to conventional and octagonal fibers.
\end{abstract}

Keywords: modal noise, annealed fiber, near infrared, radial velocity, spectroscopy

\section{INTRODUCTION}

Of the nearly 1500 planets confirmed around stars other than our own, roughly a third of these have been found by observing periodic variations in the host star's radial velocity via the Doppler shift in the star's spectrum. Of those within $100 \mathrm{Pc}$, over $90 \%$ of exoplanet discoveries are attributed to radial velocity observations. ${ }^{1}$ The use of radial velocity observations for finding planets around main sequence stars could not have proceeded without the simultaneous development of high stability, high resolution spectrographs and of high precision wavelength calibration sources such as the thorium argon (Th-Ar) lamp and the iodine (I2) cell. As the search for extrasolar planets moves towards ever lower mass planets at larger distances from their host stars, greater precision is required, placing an ever increasing burden on the calibration systems used to support these high precision devices. This, in turn, motivates increased attention to sources of noise in these calibrators. In the case of fiber fed spectrographs, one area of concern is modal noise in both the calibration and science fibers. The introduction of single mode fiber based calibration sources such as laser frequency combs has further exacerbated the modal noise issues when coupled to multimode calibration fibers.

We explore the performance of a novel approach to mode scrambling using annealed multimode fiber and compare the near field scrambling characteristics of annealed fiber with and without agitation to conventional multimode fiber, octagonal fiber and to integrating sphere scrambling. The tests in this paper were performed using a coherent, monochromatic NIR source to simulate one mode of a laser frequency comb.

One significant restriction on the ultimate precision achievable by fiber fed calibration systems is the modal noise observed in multimode fibers. Modal noise arises from an unstable population of modes in an optical fiber. At wavelength $\lambda$ a circular optical fiber of diameter $d$ and numerical aperture NA can support a finite number of modes:

$$
N=0.5\left(\frac{\pi d N A}{\lambda}\right)^{2}
$$

* steven.osterman@jhuapl.edu; Johns Hopkins University Applied Physics Laboratory, 11100 Johns Hopkins Rd, Laurel, MD 20723; phone: 1 240-228-2754 
If a subset of these modes are excited at the input end of an optical fiber, the distribution of modes can change as a result of movement, distortion or a change in stresses in the fiber, resulting in a change in the near and far field illumination patterns at the output of the fiber. This results in a change in the apparent line spread function of the spectrograph due to changing the distribution of light at the entrance aperture (near field) and to changes in the illumination of optical elements throughout the spectrograph (far field). This problem is further complicated by the introduction of single mode fiber calibrators such as laser frequency comb systems that must then be coupled into a multimode fiber. ${ }^{2,3,4}$

Other issues that arise from significant mode redistribution include light loss and focal ratio degradation. These are of considerable concern in the case of the astronomical signal coupled in from a telescope, but are less of an issue in the case of laser based reference sources, which may be orders of magnitude brighter than needed for calibration purposes.

Approaches to minimizing modal noise include fiber stressing, double scrambling and agitation. In addition, non-circular fibers have been studied, ${ }^{5,6}$ as well as the use of an integrating sphere coupled with a holographic diffuser to vary the mode distribution spatially and temporally. ${ }^{7}$ We add to this effort by exploring the performance of annealed fiber as a mode scrambler. ${ }^{8}$ An integrating sphere combined with a broadband incoherent source is used as the baseline for this study.

\section{EXPERIMENT DESCRIPTION}

\subsection{Test objectives and configuration}

The objective of our effort was to explore ways to mitigate modal noise in a system similar to the frequency comb calibrator, where coherent light from the comb is coupled from a single mode fiber into a multimode fiber. We restrict the tests to simulate a calibration system where coherent light from a single mode fiber is used to illuminate a multimode fiber such as might be used in a high precision spectrograph.

In place of the comb we use a $\mathrm{CW}$ laser operating at $1.55 \mu \mathrm{m}$. Light from the laser is injected into a $4 \mathrm{~m}$ length of single mode fiber. The single mode fiber is used to feed one of several test elements: A $4 \mathrm{~m}$ segment of $300 \mu \mathrm{m}$ (multimode) step index optical fiber (Polymicro FI 0.22 NA low OH fiber), an $8 \mathrm{~m}$ segment of $200 \mu \mathrm{m}$ octagonal fiber (CeramOptec Optran NCC fused silica core fiber, described in Plavchan, 2013), two different annealed fiber segments made from 300 $\mu \mathrm{m}$ step index Polymicro type FI multimode fiber, or an integrating sphere. These are then coupled to another length of $300 \mu \mathrm{m}$ multimode fiber, and the output face of this final fiber is imaged onto an InGaAs IR detector (figure 1).

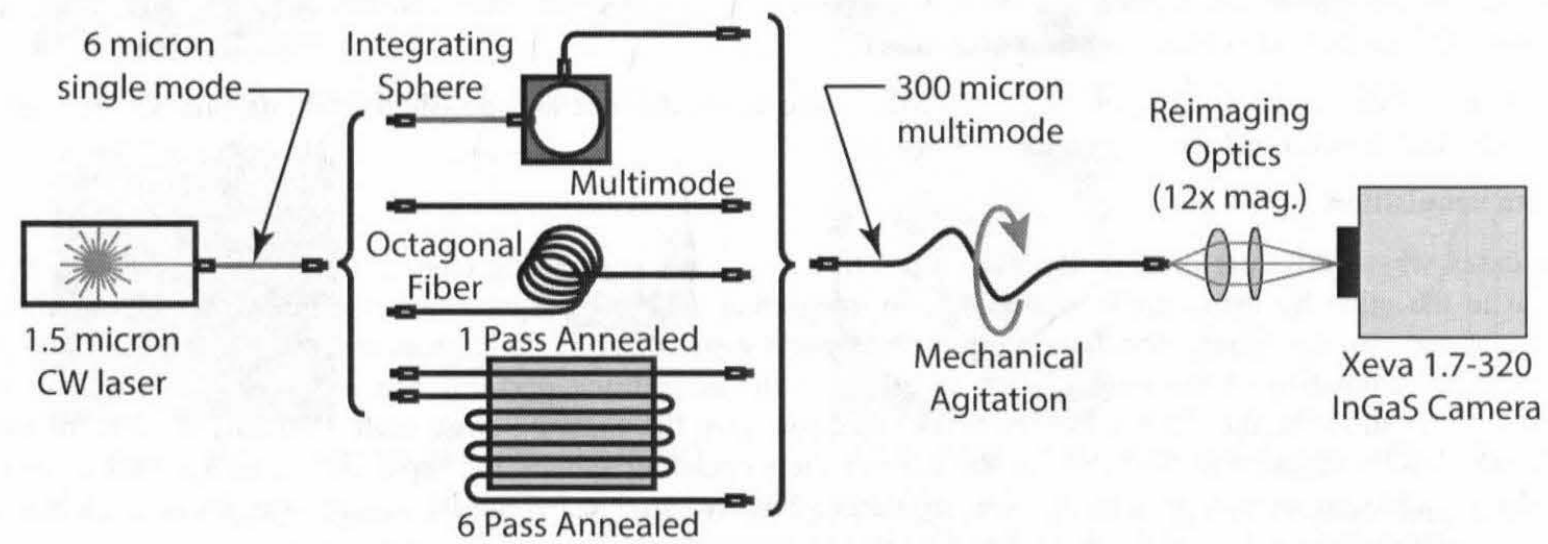

Figure 1. Near field modal noise test setup. Light from a $1.55 \mu \mathrm{m} \mathrm{CW}$ laser is delivered to a $6 \mu \mathrm{m}$ single mode fiber. The fiber is then connected to either a single $4 \mathrm{~m}$ segment of $300 \mu \mathrm{m}$ multimode fiber, an integrating sphere, an $8 \mathrm{~m}$ segment of $200 \mu \mathrm{m}$ core octagonal fiber, a single pass annealed fiber segment or a six pass annealed fiber segment. Output from the test segment is then mated to a $300 \mu \mathrm{m}$ core multimode fiber which could be agitated mechanically. The output of the final multimode segment is reimaged onto an InGaAs sensor. The integrating sphere input fiber could also be agitated, and as a baseline the integrating sphere was also illuminated with an incandescent infrared source to explore stability of the test configuration. 


\subsection{Fiber annealing procedure}

Annealing of optical fiber leads to devitrification and can be achieved by heating the fiber to between 850 and $1300^{\circ} \mathrm{C}$. This process induces growth of crystalline structures starting at the surface of the fiber and moving inwards and can result in loss of transmission in long sections of fiber, but can induce mode scrambling in shorter annealed segments. A good discussion of the annealing process can be found in Rose, 1997. ${ }^{9}$ To create our annealed fiber scrambler we limited our peak temperature to $1100^{\circ} \mathrm{C}$, a temperature high enough that Rose observed surface layer crystallization but not so high as to allow the crystal formation to propagate throughout the thickness of the fiber.

To prepare for the annealing process of the fibers, $300 \mu \mathrm{m}$ multimode fiber was threaded through a 48 inch long quartz tube with aluminum end caps. Each end cap had nine evenly spaced holes through which the optical fiber was threaded. A single length of fiber was used to create a continuous segment passing six times through the annealing tube. In addition, the tube held three single pass fibers. The fiber ends were left untreated. The fibers were pulled tightly and glued at the end caps to keep them taut and not touching each other (figure 2).

The fiber plus tube assembly was placed in a Lindburg/Blue Mini-Mite tube furnace. The fiber ends and loops of fibers on each end of the tube were supported at the level of the tube to relieve any stress on the fibers. The tube extended through the furnace with the central portion of the tube being heated, permitting us to process just the central $30 \mathrm{~cm}$ of each fiber segment.

The tube furnace was programmed to heat at the rate of $10^{\circ} \mathrm{C} / \mathrm{min}$ until it reached a peak temperature of $1100^{\circ} \mathrm{C}$. This temperature was maintained for four hours, at which time the tube furnace began its cooling cycle programmed at a rate of $20^{\circ} \mathrm{C} / \mathrm{min}$; however, the actual temperature drop was significantly slower rate than the programmed rate, falling only about $5^{\circ} \mathrm{C} / \mathrm{min}$.

During the annealing process the outer polyimide cladding burned off, leaving some ash residue on the fibers and the fibers slightly elongated and sagged. To prevent the fibers from contacting one another, the end caps were carefully extended a few millimeters outward and fixed in place.

Once the annealing process was complete, the fibers were connectorized and polished, creating one six pass and three single pass segments for testing.

\subsection{Data acquisition}

In the cases where different optical fibers are used to achieve the scrambling (figure 1), light was coupled from one segment to the other by direct contact coupling. In integrating sphere configurations, the input and output fibers were simply inserted into the sphere. Input and output fibers ports were offset by 90 degrees and separated by a baffle plate to avoid direct illumination of the output fiber. In all cases the scrambling performance was observed with and without mechanical agitation. In the fiber only cases, the agitation took the form of large scale $(\sim 10 \mathrm{~cm})$ shaking of the postsample multimode segment at $10-20 \mathrm{~Hz}$. In the case of the integrating sphere, the input fiber was agitated at several $\mathrm{Hz}$ manually in addition to post scrambler fiber agitation. Finally, an incoherent IR source was used to illuminate the integrating sphere entrance to establish the baseline image centroid stability.

\section{RESULTS}

A series of 32 images was observed for each configuration at $0.1 \mathrm{~ms}$ and $100 \mathrm{~ms}$ frame rates (1 second for integrating sphere configurations), along with background and flat field images. Single unagitated fast frame images and $3.2 \mathrm{sec}$ ( 32 second for integrating sphere sets) accumulated agitated images are shown in figure 3 and 4 .

\footnotetext{
Specific manufacturer or part number information does not imply endorsement but is provided for completeness.
} 

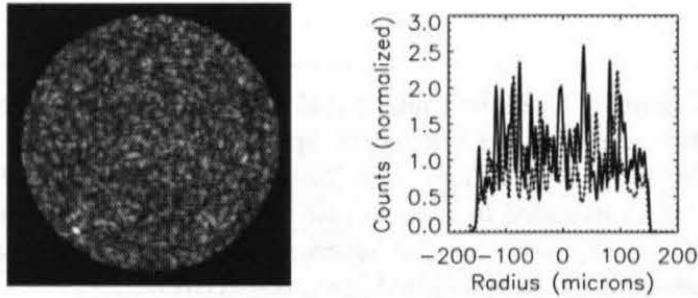

Multimode, single frame ( $0.1 \mathrm{~ms}$ static)
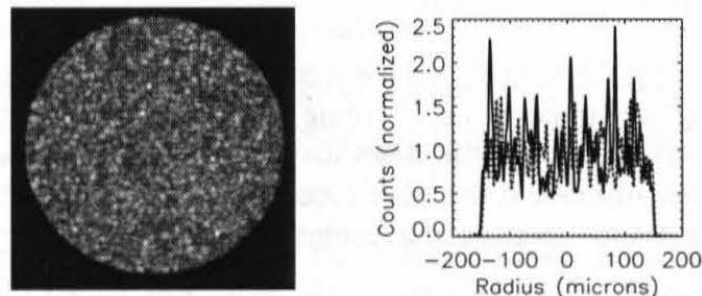

Octagonal fiber, single frame (0.1 ms static)
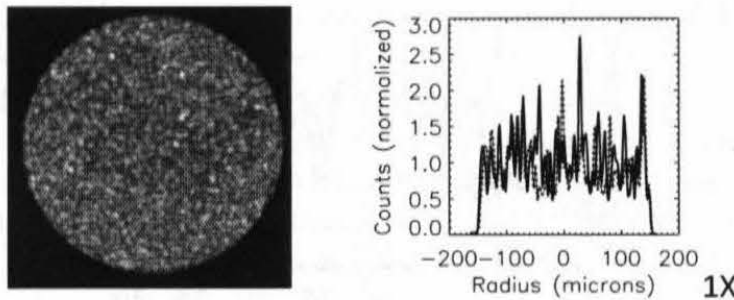

Annealed, single frame (0.1 ms static)
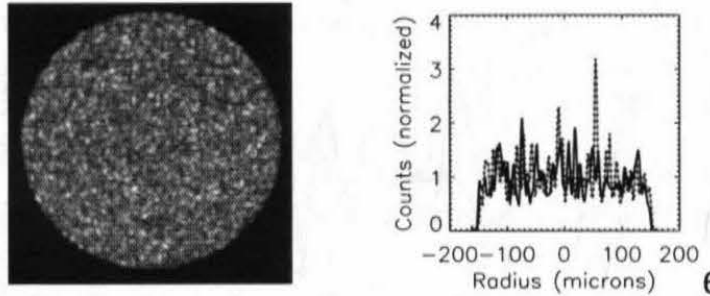

Annealed, single frame (0.1 ms static)
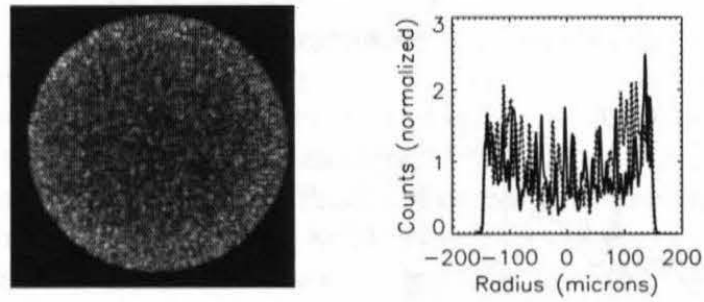

Integrating sphere, single frame (1 s static)
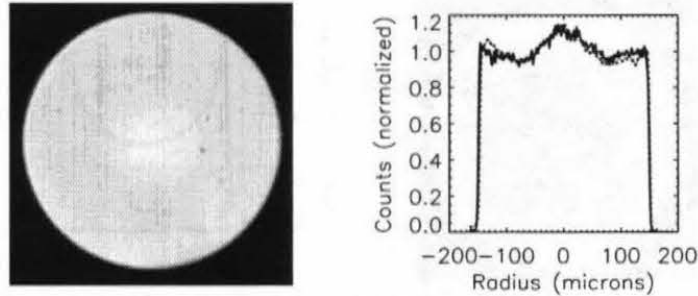

Multimode, agitated, $3.2 \mathrm{sec}$ integration
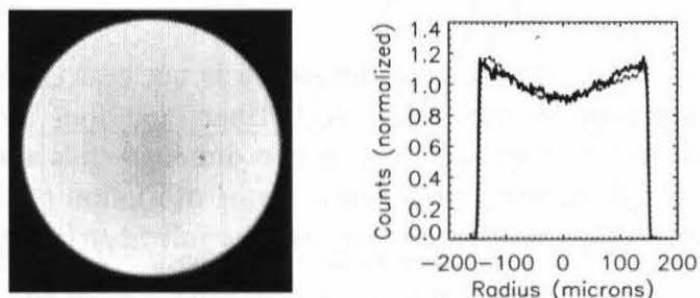

Octagonal fiber, agitated, $3.2 \mathrm{sec}$ integration
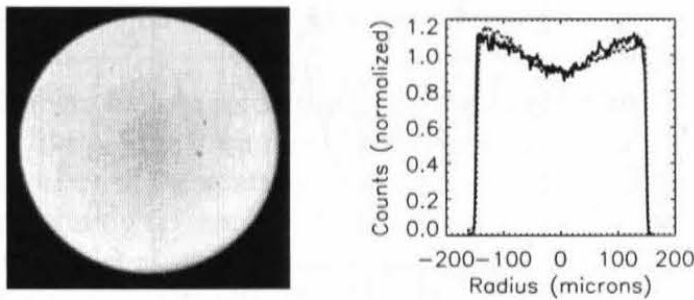

1X Annealed, agitated, $3.2 \mathrm{sec}$ integration
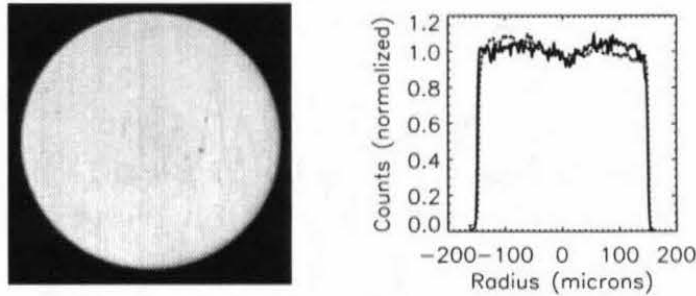

6X Annealed, agitated, 3.2sec integration
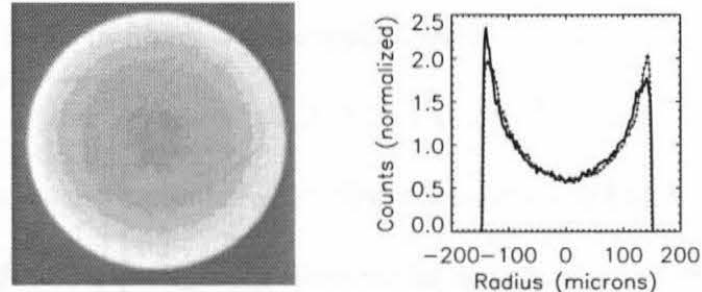

Integrating sphere, agitated, $32 \mathrm{sec}$ integration

Figure 3. Near field images for all test cases, both for a static image with no agitation and for extended integrations with mechanical agitation. Agitation occurred in a segment of multimode fiber following the test element. In the case of the integrating sphere the agitation also occurred at the single mode to sphere interface. In all cases agitation is clearly required to achieve an acceptable level of uniformity. $\mathrm{X}$ and $\mathrm{Y}$ axis single pixel slices along the center of the images are shown in solid and dotted lines, respectively. 

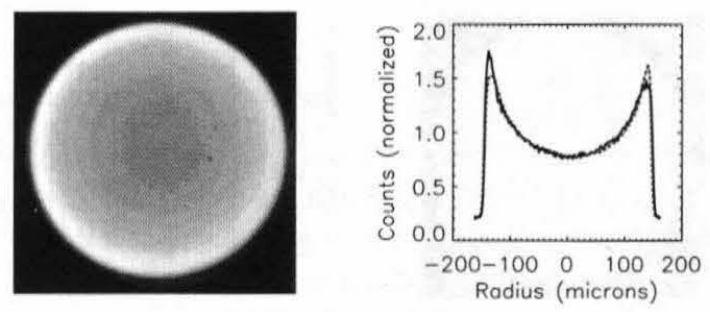

Integrating sphere, IR source
Figure 4. Near field image and radial distributions along two axes for the integrating sphere with an incoherent, broadband IR source. This case shows greatest stability and is included to demonstrate the minimum RV motion achievable with the test setup. X and $\mathrm{Y}$ central cuts are shown in solid and dotted lines, respectively.

In all cases the most striking difference is not between scrambling techniques, but between static single frames and the long integration images taken with fiber agitation, indicating the importance of some sort of dynamic scrambling. Variation in the image centroid in two dimensions is shown in figure 6, and the RMS displacements are summarized in table 1. Displacements are given in terms of motion of the center of mass at the fiber face, and in terms of RV variation for a hypothetical instrument that uses the full fiber face as the entrance aperture at a resolution of 70,000 .

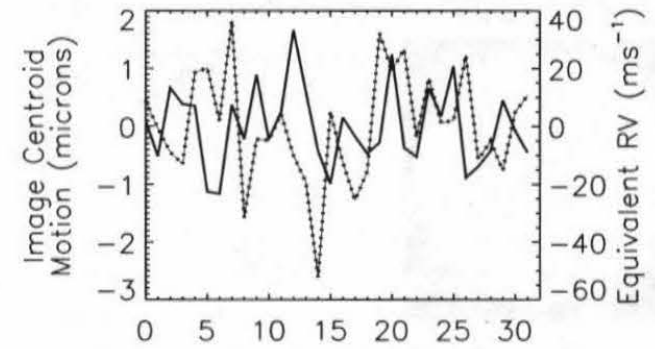

Multimode Fiber only

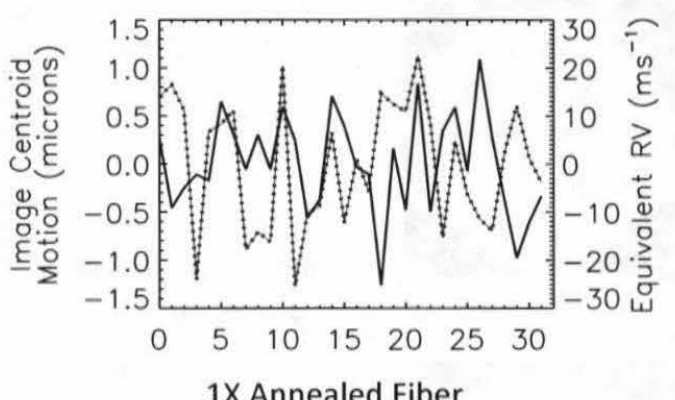

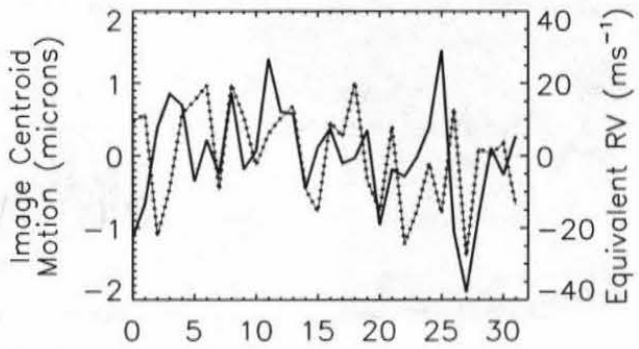

Octagonal Fiber

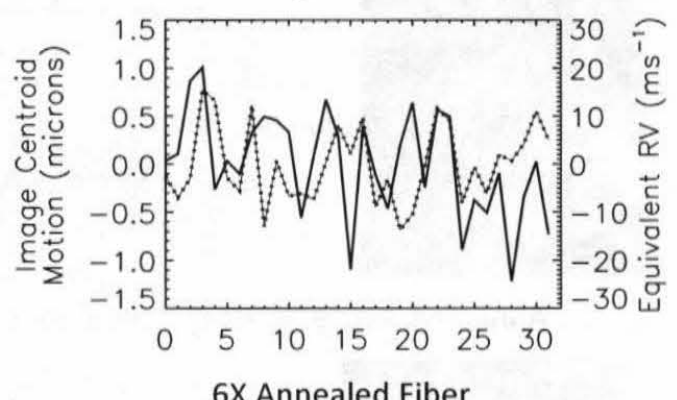

6X Annealed Fiber

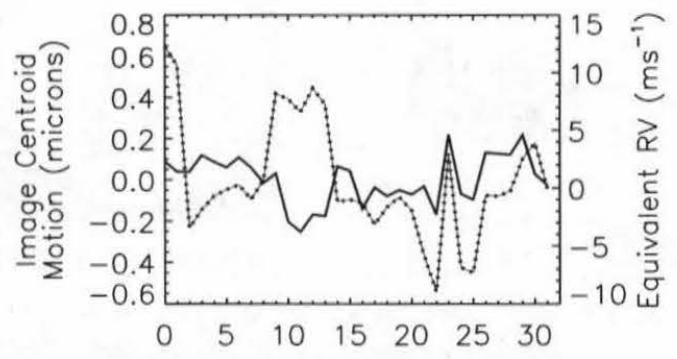

Integrating Sphere (coherent source)

Figure 5. Near field image centroid motion in microns at the fiber face and in $\mathrm{ms}^{-1} \mathrm{RV}$ equivalent. (Centroid motions along the $\mathrm{X}$ and $\mathrm{Y}$ axes are shown in solid and dotted lines, respectively). RMS motions are summarized in table 1. 


\begin{tabular}{|l|c|}
\hline Test case & $\begin{array}{c}\text { RMS Radial Centroid } \\
\text { Displacement }\end{array}$ \\
\hline Multimode Fiber only $(0.1 \mathrm{~ms}$ frames) & $1.17 \mu \mathrm{m}\left(16.8 \mathrm{~ms}^{-1}\right)$ \\
\hline Octagonal Fiber $(0.1 \mathrm{~ms}$ frames) & $0.996 \mu \mathrm{m}\left(14.2 \mathrm{~ms}^{-1}\right)$ \\
\hline 1X Annealed Fiber $(0.1 \mathrm{~ms}$ frames) & $0.851 \mu \mathrm{m}^{-1}\left(12.2 \mathrm{~ms}^{-1}\right)$ \\
\hline 6X Annealed Fiber $(0.1 \mathrm{~ms}$ frames) & $0.682 \mu \mathrm{m}\left(9.74 \mathrm{~ms}^{-1}\right)$ \\
\hline Integrating Sphere with Agitated Input $(1.0 \mathrm{~s}$ frames) & $0.313 \mu \mathrm{m}\left(4.48 \mathrm{~ms}^{-1}\right)$ \\
\hline $\begin{array}{l}\text { Integrating Sphere with Incoherent IR Illumination (test fixture stability } \\
\text { demonstration) (1.0 s frames) }\end{array}$ & $0.022 \mu \mathrm{m}\left(0.310 \mathrm{~ms}^{-1}\right)$ \\
\hline $\begin{array}{l}\text { Table 1. Radial motion of the observed image centroid relative to the mean position in microns and RV equivalent } \\
\text { motion for six test cases. Integrating sphere integration periods were longer than fiber only integrations due to the } \\
\text { low efficiency of the sphere and so are expected to show much better scrambling. RV equivalent is provided strictly } \\
\text { for context since only the component of motion parallel to the dispersion axis would directly contribute to RV error. }\end{array}$ \\
\hline
\end{tabular}

From these results it appears that octagonal fibers and annealed fibers have a consistent advantage over single mode fiber alone, and that the annealed fibers outperform the octagonal fiber. The improved scrambling of octagonal fibers is qualitatively consistent with previous studies. ${ }^{5,6}$ While the differences in integration time make any direct comparison in this study problematic, the excellent results obtained with the integrating sphere are consistent with Mahadevan et al. ${ }^{7}$ An update to the technique used in [7] is provided in papers by Halverson et al. and Roy et al. in these proceedings. ${ }^{10,11}$

\section{ACKNOWLEDGEMENTS}

Research presented in this paper was supported by NSF grants AST-0906034, AST-1126413, and AST-131088.

\section{REFERENCES}

[1] Wright, J., and Marcy, G., "Exoplanet Orbit Database,” [Online], available: <http://www.Exoplanets.org >.

[2] Baudrand, J., and Walker, G., "Modal Noise in High-Resolution, Fiber-fed Spectra: A Study and Simple Cure," PASP, 113, 851-858 (2001).

[3] Wood, T., "Actual modal power distributions in multimode optical fibers and their effect on modal noise," Optics Lett., 9, 102-104 (1984).

[4] Goodman, J. W., and Rawson, E. G., "Statistics of modal noise in fibers: A case of constrained speckle," Optics Lett., 6, 324-326 (1981).

[5] Spronck, J. F. P., Kaplan, Z. A., Fischer, D. A., Schwab, C., and Szymkowiak, A. E., "Extreme Doppler precision with octagonal fiber scramblers," Proc. SPIE 8446, 84468T-1 -10 (2012).

[6] Plavchan, P. P., et al., "Precision near-infrared radial velocity instrumentation II: Non-Circular Core Fiber Scrambler," Proc. SPIE 8864, 88640G-1 -18 (2013).

[7] Mahadevan, S., Halverson, S., Ramsey, L., and Venditti, N., "Suppression of fiber modal noise induced radial velocity errors for bright emission-line calibration sources," ApJ, 786, 18-24 (2014).

[8] Schlager, J. B., and Rose, A. H., “Annealed Optical Fiber Mode Scrambler,” Elec. Lett., 37, 9-10 (2001).

[9] Rose, A. H., "Devitrification in Annealed Optical Fiber," J. Lightwave Tech., 15, 808-814 (1997).

[10] Halverson, S., et al. "The habitable-zone planet finder calibration system," Proc. SPIE 9147 (in press).

[11] Roy, A., Halverson, S., Mahadevan, S., and Ramsey, L. W., "Scrambling and modal noise mitigation in the habitable zone planet finder fiber feed," Proc. SPIE 9147 (in press). 